\title{
A renormalizable supersymmetric $\mathrm{SO}(10)$ model with natural doublet-triplet splitting
}

\author{
Ying-Kang Chen and Da-Xin Zhang \\ School of Physics and State Key Laboratory of Nuclear Physics and Technology, Peking University, \\ Yiheyuan Road, Beijing 100871, China \\ E-mail: ykchen@pku.edu.cn, dxzhang@pku.edu.cn
}

ABSTRACT: We propose a renormalizable supersymmetric SO(10) model where the doublettriplet splitting problem is solved using the Dimopoulos-Wilczek mechanism. An unwanted coupling is forbidden through a filter sector. To suppress proton decay without spoiling gauge coupling unification, there is a problem in the weak doublets which requires further improvements.

Keywords: GUT, Beyond Standard Model, Gauge Symmetry

ArXiv EPRINT: 1410.5625 


\section{Contents}

1 Introduction 1

2 A model without $120 \quad 2$

3 The massless states in a model with $120 \quad 4$

4 The filter sector for DW mechanism in the presence of $120 \quad 5$

$\begin{array}{llr}5 & \text { The full model } & 8\end{array}$

$\begin{array}{lll}6 & \text { Summary } & 9\end{array}$

$\begin{array}{ll}\text { A The full mass matrix for the weak doublets } & 10\end{array}$

\section{Introduction}

Grand Unified Theories (GUTs) are very important candidates for the physics beyond the Standard Model (SM). In GUT models Higgs fields are unified into complete multiplets of the GUT symmetry. Consequently, a serious problem in GUT models is that while the weak doublets are massless compared to the GUT scale, the color-triplets in the same multiplets are very heavy as required by proton decay experiments. This problem is eased in the supersymmetric (SUSY) versions of GUT models where if this doublet-triplet splitting (DTS) is realized at tree level, the non-renormalization theorem protects the DTS from radiative corrections.

In SUSY SO(10) models [1, 2], to solve the DTS problem several mechanisms have been developed [3-18], among which the Missing Vacuum Expectation Value (Missing VEV) or the Dimopoulos-Wilczek (DW) mechanism [6-18] is widely used. It uses the adjoint Higgs superfield in $\mathbf{4 5}$, usually assisted by Higgs in $\mathbf{5 4}$, to break $\mathrm{SO}(10)$ down to $\mathrm{SU}(3)_{c} \times \mathrm{SU}(2)_{L} \times \mathrm{U}(1)_{R} \times \mathrm{U}(1)_{B-L}$ (or to $\mathrm{SU}(3)_{c} \times \mathrm{SU}(2)_{L} \times \mathrm{SU}(2)_{R} \times \mathrm{U}(1)_{B-L}$ when the DW mechanism applies). Since the $\mathbf{4 5}$ is anti-symmetric, two different Higgs in $\mathbf{1 0}$ $\left(\mathbf{1 0}_{1,2}\right)$ are needed to couple with $\mathbf{4 5}$. When the $\mathbf{4 5}$ acquires a VEV only in the $(15,1,1)$ but not in the $(1,1,3)$ direction, where the numbers in the brackets are representations under the $\mathrm{SU}(4)_{C} \times \mathrm{SU}(2)_{L} \times \mathrm{SU}(2)_{R}$ subgroup of $\mathrm{SO}(10)$, all the color-triplets are massive while the weak doublets can be massless.

To further break down the symmetry into the SM gauge group, representations containing $B-L$ breaking VEVs are needed. In the non-renormalizable models, Higgs in $\mathbf{1 6}+\overline{\mathbf{1 6}}$ are introduced. This will bring into the dangerous dimension- $4 R$-parity violating operators which lead to too rapid proton decay rates. Also, to describe correct fermion masses, operators of higher dimensions are used which make the models have less predictive abilities. 
In the renormalizable models, we need Higgs in $\mathbf{1 2 6}+\overline{\mathbf{1 2 6}}$ [19], usually even accompanied by 120 [20-31], to describe fermion masses. The DW mechanism is mostly realized in the non-renormalizable models [11-18], except in [10] which is (partially) renormalizable.

In the renormalizable model of [10], the key point is to introduce the couplings $\overline{\mathbf{1 2 6}} \cdot \mathbf{2 1 0} \cdot \mathbf{1 2 6}$ and $10_{1} \cdot \mathbf{2 1 0} \cdot \mathbf{1 2 6}$ while the coupling $10_{1} \cdot \mathbf{2 1 0} \cdot \overline{\mathbf{1 2 6}}$ is absent. This is realized by enforcing an extra symmetry. An unsatisfactory aspect in the model of [10] is the introduction of explicit symmetry breaking terms in the model. Also, the transformation properties of $\mathbf{1 0}_{\mathbf{1}}$ and $\overline{\mathbf{1 2 6}}$ are different under this extra symmetry, so that they cannot couple with the matter fields at the same time. Then the non-renormalizble Yukawa couplings are inevitable to account for correct fermion masses. Furthermore, $\mathbf{1 2 0}$ cannot be incorporated easily in the model since the coupling $\mathbf{1 2 0} \cdot \mathbf{4 5} \cdot \mathbf{1 0}_{\mathbf{2}}$, which breaks the DW mechanism, generally exists, if the $\mathbf{1 2 0}$ has the same transformation property as the $\mathbf{1 0}_{\mathbf{1}}$ under the extra symmetry.

In this paper, our aim is limited to present a renormalizable model to realize the DW mechanism. Comparing with [10] in which the only renormalizable Yukawa couplings are those with Higgs in $\mathbf{1 0}_{1}$, we can include renormalizable Yukawa couplings with $\overline{\mathbf{1 2 6}}$ and even with 120, so that fermion masses are fully realistic. However, we will not fully solve the problem on proton decay which needs mechanism [32] other than the DW mechanism, although the proton decay amplitudes mediated by the color triplets in $\mathbf{1 0}_{\mathbf{1}}$ are suppressed without spoiling gauge coupling unification. We will first present a renormalizable model without 120, next include $\mathbf{1 2 0}$ resulting a pair of massless doublets and a pair of massless triplets, then propose a method to give masses to the triplets using the DW mechanism by introducing a "filter sector" to forbid the unwanted $\mathbf{1 2 0} \cdot \mathbf{4 5} \cdot \mathbf{1 0}_{\mathbf{2}}$ coupling, and give a full model. We will finally summarize.

\section{A model without 120}

In order to clarify how to implement the DW mechanism in renormalizable models to realize the DTS, we present a model without 120 firstly. The field content consists three $45\left(A, A^{\prime}, A^{\prime \prime}\right)$, two $\mathbf{5 4}\left(S, S^{\prime}\right)$, one pair of $\mathbf{1 2 6}+\overline{\mathbf{1 2 6}}(\Delta$ and $\bar{\Delta})$, one $\mathbf{2 1 0}(\Phi)$, and two 10s $\left(H_{1,2}\right)$. One $\mathrm{SO}(10)$ singlet $(Y)$ is also introduced whose VEV acts as the mass for the first two 45s $\left(A, A^{\prime}\right)$ which are to acquire DW VEVs. The third $45\left(A^{\prime \prime}\right)$ is used to eliminate redundant Goldstones $[8,9]$. The first $\mathbf{5 4}(S)$ is used to generate the DW VEVs, while the second $\mathbf{5 4}\left(S^{\prime}\right)$ is required to maintain SUSY. $\Delta$ and $\bar{\Delta}$ are used to reduce the rank of gauge group. 210 is used in mixing $H_{1}$ and $\Delta$.

We impose a $\mathbb{Z}_{12}$ symmetry. Transformation properties of all the Higgs superfields are listed in table I, besides the matter superfields of all the three generations whose $\mathbb{Z}_{12}$ charges are taken as -1 . The most general Higgs superpotential is

$$
W=W_{D W}+W_{S B}+W_{D T},
$$

where

$$
W_{D W}=Y A A^{\prime}+S A A^{\prime}+A A^{\prime} A^{\prime \prime}
$$




\begin{tabular}{|c|c|c|c|c|c|c|c|c|c|c|c|}
\hline & $H_{1}$ & $H_{2}$ & $\bar{\Delta}$ & $\Delta$ & $\Phi$ & $A$ & $A^{\prime}$ & $A^{\prime \prime}$ & $S$ & $S^{\prime}$ & $Y$ \\
\hline $\mathbb{Z}_{12}$ & 2 & 6 & 2 & -2 & 0 & 4 & 2 & 6 & 6 & 0 & 6 \\
\hline
\end{tabular}

Table 1. A renormalizable model without $\mathbf{1 2 0}$ with a $Z_{12}$ symmetry imposed. A field $X$ with charge $q$ transforms as $X \rightarrow X e^{i q \pi / 6}$.

$$
\begin{aligned}
W_{S B}= & \frac{1}{2} m_{\Phi} \Phi^{2}+\Phi^{3}+\Phi A^{\prime \prime 2}+\frac{1}{2} m_{A^{\prime \prime}} A^{\prime \prime 2}+\frac{1}{2} m_{Y} Y^{2} \\
& +\frac{1}{2} m_{S} S^{2}+\frac{1}{2} m_{S^{\prime}} S^{\prime 2}+S^{\prime 3}+S^{2} S^{\prime}+S^{\prime} \Phi^{2} \\
& +S^{\prime} A^{\prime \prime 2}+S S^{\prime} A^{\prime \prime}+S S^{\prime} Y \\
W_{D T}= & \frac{1}{2} m_{2} H_{2}^{2}+S^{\prime} H_{2}^{2}+H_{1} A H_{2}+\left(H_{1}+\bar{\Delta}\right) \Phi \Delta+m_{\Delta} \Delta \bar{\Delta} .
\end{aligned}
$$

Here we have suppressed all dimensionless couplings for simplicity. In (2.3) we do not include the nonrenormalizable couplings such as $\frac{1}{\Lambda} H_{1} H_{1} A A$ which will otherwise break the DW mechanism of realizing the DTS. In the nonrenormalizable models, such higher dimensional couplings must be considered because other couplings of the same properties are used. However, in the renormalizable models, we can simply regard whether these couplings exist or not depend on if there are states of suitable representations which can generate the relevant tree-level diagrams. Here, loop diagrams are not important, as they are SUSY breaking effects which are small. Note that even in the nonrenormalizable models, how large the higher dimensional couplings are is not known [9].

In $W_{D W}$, the first two terms are the modified form for realization of the DW mechanism [9], while the third term, which has no effect on the F-flatness conditions of keeping SUSY at high energy, is introduced to eliminate extra Goldstone particles in $A$ and $A^{\prime}[8]$.

Under $\mathrm{SU}(4)_{C} \otimes \mathrm{SU}(2)_{L} \otimes \mathrm{SU}(2)_{R}$, there are possibly two VEVs for every 45, e.g. $A_{1}$ and $A_{2}$ for $A$ in the $(1,1,3)$ and $(15,1,1)$ directions, respectively. Setting the F-terms of $A_{1}^{\prime}$ and $A_{2}^{\prime}$ to zeros, we get the following equations

$$
0=A_{1}\left(Y+\frac{3}{2 \sqrt{15}} S\right), 0=A_{2}\left(Y-\frac{1}{\sqrt{15}} S\right) .
$$

Hereafter we will use the same symbols for the fields and their VEVs. One set of the solutions are

$$
A_{1}=0, \quad A_{2} \neq 0, \quad Y=\frac{1}{\sqrt{15}} S,
$$

which realize the DW VEVs. It also follows $A_{1}^{\prime}=0$ and $A_{2}^{\prime} \neq 0$ when the F-flatness conditions for $A$ are enforced.

Notice that using the DW VEV, $A_{1}=0$, the doublets in $H_{2}$ separate from the other Higgs doublets. Then the up-type doublet in $\Delta$ acquires masses only through its couplings with the down-type doublets in $H_{1}$ and in $\bar{\Delta}$. To be explicitly, the mass matrix for the weak doublets is

$$
\left(\begin{array}{c|cc}
0_{2 \times 2} & A_{11} & 0 \\
& A_{21} & 0 \\
\hline B_{2 \times 2} & C_{2 \times 2}
\end{array}\right),
$$


where the columns are ordered as $\left(H_{1}^{u}, \bar{\Delta}^{u} ; \Delta^{u}, \Phi^{u}\right)$ and the rows are $\left(H_{1}^{d}, \bar{\Delta}^{d} ; \Delta^{d}, \Phi^{d}\right)$. Expressions for individual elements can be found in [33, 34].

(2.5) has a zero eigenvalue. To be more explicitly, a combination of the first two rows gives a new row with all its elements to be zeros, and the corresponding combination of $H_{1}^{d}$ and $\bar{\Delta}^{d}$ is identified as the $H_{d}^{0}$ of the Minimal Supersymmetric Standard Model (MSSM). There also exists a massless up-type Higgs doublet $H_{u}^{0}$ of the MSSM, since the first, the second and the last columns in (2.5) can give a column with all its elements to be zeros so that the massless $H_{u}^{0}$ has components from $H_{1}^{u}, \bar{\Delta}^{u}$ and $\Phi^{u}$. The above arguments do not apply for the color-triplet sector due to the coupling $H_{1} A H_{2}$ where $A_{2} \neq 0$ couples these triplets so that $\mathrm{H}_{2}$ does not separate from other Higgs in the triplet sector.

The above observation follows [10] directly. The key point is the presence of $H_{1} \Delta \Phi$ and the absence of $H_{1} \bar{\Delta} \Phi$, otherwise two or null zero eigenvalues exist in the doublet mass matrix. The differences from [10] are that the present model is fully consistent with the imposed symmetry, and $H_{1}$ and $\bar{\Delta}$ have the same transformation properties under this symmetry so that they can couple with matter fields simultaneously.

\section{The massless states in a model with 120}

In the presence of $\mathbf{1 0}, \mathbf{1 2 0}, \overline{\mathbf{1 2 6}}, \mathbf{1 2 6}, \mathbf{2 1 0}$, we need to construct a model which contains a pair of massless weak doublets and a pair of massless color triplets while keeping all other states massive. After realizing the DW mechanism which will be performed in the next section, the color triplets will get masses while the weak doublets keep massless.

We will introduce two $\mathbf{1 2 0}\left(D_{1,2}\right)$ and the relevant superpotential is

$$
\left(H_{1}+D_{1}+\bar{\Delta}\right) \Phi\left(\Delta+D_{2}\right)+m_{D} D_{1} D_{2}+m_{\Delta} \Delta \bar{\Delta}+\frac{1}{2} m_{\Phi} \Phi^{2}+\Phi^{3} .
$$

Note from (3.1) all the states in $D_{1,2}, \bar{\Delta}, \Delta$, $\Phi$ get masses. The only possible massless states are the doublets and triplets in $10_{1}$. Now we exam if there are still massless states in the presence of the interactions (3.1) which mix all the doublets and triplets.

The contents of all doublets, besides the pair from $\mathrm{H}_{2}$ which decouple, are

- one pair from $H_{1}\left(H_{1}^{u}+H_{1}^{d}\right)$;

- one pair from $\bar{\Delta}\left(\bar{\Delta}^{u}+\bar{\Delta}^{d}\right)$;

- one pair from $\Delta\left(\Delta^{u}+\Delta^{d}\right)$;

- two pairs from each of $D_{i=1,2}\left(D_{i}^{u}+D_{i}^{d}\right.$ and $\left.D_{i}^{\prime u}+D_{i}^{\prime d}\right)$. The unprimed and primed doublets are in $(1,2,2)$ and $(15,2,2)$ under $\mathrm{SU}(4)_{C} \otimes \mathrm{SU}(2)_{L} \otimes \mathrm{SU}(2)_{R}$, respectively;

- one pair from $\Phi\left(\Phi^{u}+\Phi^{d}\right)$.

From (3.1), the mass matrix for the doublets is

$$
\left(\begin{array}{c|c}
0_{4 \times 4} & A_{4 \times 3} 0_{4 \times 1} \\
\hline B_{4 \times 4} & C_{4 \times 4}
\end{array}\right),
$$


where the columns are ordered as $\left(H_{1}^{u}, D_{1}^{u}, D_{1}^{\prime u}, \bar{\Delta}^{u} ; \Delta^{u}, D_{2}^{u}, D_{2}^{\prime u}, \Phi^{u}\right)$ and the rows by replacing the superscripts " $u$ " by "d". The matrices $B$ is fully ranked, $C$ is not fully null while most of elements in $A$ are nonzero. The key ingredient is the $0_{4 \times 1}$ sub-matrix as a consequence of the absence of the coupling $H_{1} \Phi \bar{\Delta}$ which, if present, will induce a nonzero $4 \times 1$ matrix with elements proportional to the VEV of $\bar{\Delta}$. It is clear that there is a zero eigenvalue in (3.2); or more explicitly, since a combination of the first four rows in (3.2) can give a row with all its elements to be zeros, a combination of $H_{1}^{d}, D_{1}^{d}, D_{1}^{\prime d}, \bar{\Delta}^{d}$ gives the massless eigenstate $H_{d}^{0}$ of the MSSM. Similarly, the massless eigenstate $H_{u}^{0}$ has components from the doublets in $H_{1}^{u}, D_{1}^{u}, D_{1}^{\prime u}, \bar{\Delta}^{u}$ and $\Phi^{u}$.

For the triplets, there are one more pair compared to the doublets. They are one more color-triplet from $\bar{\Delta}$ and one more color-anti-triplet from $\Delta$. Ordering the columns as $\left(H_{1}^{T}, D_{1}^{T}, D_{1}^{\prime T}, \bar{\Delta}^{T}, \bar{\Delta}^{\prime T} ; \Delta^{T}, D_{2}^{T}, D_{2}^{\prime T}, \Phi^{T}\right)$ and the rows as $\left(H_{1}^{\bar{T}}, D_{1}^{\bar{T}}, D_{1}^{\prime \bar{T}}, \bar{\Delta}^{\bar{T}} ; \Delta^{\bar{T}}, \Delta^{, \bar{T}}, D_{2}^{\bar{T}}, D_{2}^{\prime \bar{T}}, \Phi^{\bar{T}}\right)$, the mass matrix for the triplets is

$$
\left(\begin{array}{c|cc}
0_{4 \times 5} & A_{4 \times 3} & 0_{4 \times 1} \\
\hline B_{5 \times 5} & C_{5 \times 4}
\end{array}\right),
$$

again, there is a pair of massless color triplets which contain nonzero components from $H_{1}^{T}$ and $H_{1}^{\bar{T}}$, respectively. In the next section, this pair of triplets will be given masses through the DW mechanism while the doublets are keeping massless.

\section{The filter sector for DW mechanism in the presence of 120}

In renormazible SUSY SO(10) models, usually a Higgs in $\mathbf{1 2 0}$ is also needed to couple with the fermion sector through Yukawa interactions $[23,29,31]$. This requires that $D_{1}$ has the same symmetry property as $H_{1}$ and $\bar{\Delta}$. When the triplets of $H_{1}$ receive masses through the coupling $H_{1} A H_{2}$, the DW VEV $A_{1}=0$ leads the doublets in $H_{1}$ to be massless, However, the coupling $D_{1} A H_{2}$ is also allowed. Consequence of this coupling $D_{1} A H_{2}$ is that the doublets in $H_{2}$ do not decouple from the other doublets due to the mixing terms

$$
-\frac{i \sqrt{2} A_{2}}{3}\left(H_{2}^{d} D^{\prime u}-D^{\prime d} H_{2}^{u}\right)
$$

in the mass matrix, which leaves no massless states in the weak doublets. Thus direct application of the DW mechanism in the presence of $\mathbf{1 2 0}$ does not realize natural DTS.

To suppress the coupling $D_{1} A H_{2}$ when $H_{1}$ and $D_{1}$ have the same symmetry behaviors under the extra symmetry, we introduce a pair of 10s $(h+\bar{h})$ and a singlet $(P)$ in $\mathrm{SO}(10)$ and replace $\frac{1}{2} m_{H} H_{2}^{2}\left(+S^{\prime} H_{2}^{2}\right)+H_{1} A H_{2}$ in (2.3) by the following superpotential

$$
W_{\text {filter }}=H_{1} P \bar{h}+m_{h} \bar{h} h+h A H_{2}+\frac{1}{2} m_{2} H_{2}^{2} .
$$




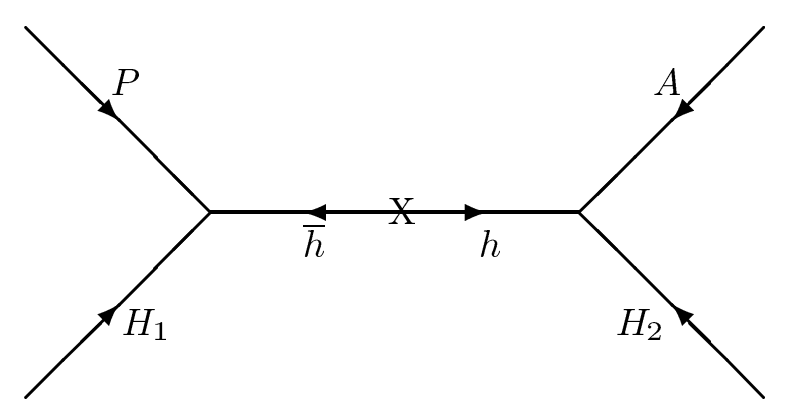

Figure 1. The filter sector which generates the $H_{1} A(P) H_{2}$ coupling but forbids the $D_{1} A(P) H_{2}$ coupling.

The mass matrix for the doublets and triplets are

$$
M_{D}=\left(\begin{array}{cccc}
0 & P & 0 & 0 \\
P & 0 & m_{h} & 0 \\
0 & m_{h} & 0 & A_{1} \\
0 & 0 & A_{1} & m_{2}
\end{array}\right)
$$

and

$$
M_{T}=\left(\begin{array}{cccc}
0 & P & 0 & 0 \\
P & 0 & m_{h} & 0 \\
0 & m_{h} & 0 & A_{2} \\
0 & 0 & A_{2} & m_{2}
\end{array}\right),
$$

respectively. The orderings in both the columns and the rows are $\left(H_{1}, \bar{h}, h, H_{2}\right)$ in (4.2), (4.3). It is now clear if the DW solutions $A_{1}=0$ and $A_{2} \neq 0$ are taken, there is a massless eigenvalue in (4.2) with the eigenstates

$$
\begin{aligned}
& H^{u}=\frac{m_{h}}{\sqrt{P^{2}+m_{h}^{2}}} H_{1}^{u}-\frac{P}{\sqrt{P^{2}+m_{h}^{2}}} h^{u}, \\
& H^{d}=\frac{m_{h}}{\sqrt{P^{2}+m_{h}^{2}}} H_{1}^{d}-\frac{P}{\sqrt{P^{2}+m_{h}^{2}}} h^{d},
\end{aligned}
$$

while no massless eigenvalue exists in the matrix $M_{T}$. This realization of the DW mechanism of giving masses to the triplets can be depicted in figure 1. The coupling $D_{1} A H_{2}$ is forbidden in the same time when the direct coupling $H_{1} A H_{2}$ is absent. Here the singlet $P$ plays the role as a "filter" which forbids the unwanted coupling.

The situation does not change much when the massless eigenstates are got from the last section and are not purely from $H_{1}$. Supposing that these states are

$$
\begin{aligned}
H_{u}^{0} & =C_{H_{1}}^{u} H_{1}^{u}+\cdots, & H_{d}^{0} & =C_{H_{1}}^{d} H_{1}^{d}+\cdots, \\
T^{0} & =D_{H_{1}}^{T} H_{1}^{T}+\cdots, & \bar{T}^{0} & =D_{H_{1}}^{\bar{T}} H_{1}^{\bar{T}}+\cdots,
\end{aligned}
$$


(4.2) and (4.3) are modified into

$$
M_{D}=\left(\begin{array}{cccc}
0 & C_{H_{1}}^{d} P & 0 & 0 \\
C_{H_{1}}^{u} P & 0 & m_{h} & 0 \\
0 & m_{h} & 0 & A_{1} \\
0 & 0 & A_{1} & m_{2}
\end{array}\right)
$$

and

$$
M_{T}=\left(\begin{array}{cccc}
0 & D_{H_{1}}^{\bar{T}} P & 0 & 0 \\
D_{H_{1}}^{T} P & 0 & m_{h} & 0 \\
0 & m_{h} & 0 & A_{2} \\
0 & 0 & A_{2} & m_{2}
\end{array}\right),
$$

respectively. Consequently, the conclusion which follows (4.2) still holds, provided that the massless doublets of the MSSM are now

$$
\begin{aligned}
& H^{u}=\frac{m_{h}}{\sqrt{\left|C_{H_{1}}^{u} P\right|^{2}+m_{h}^{2}}} H_{u}^{0}-\frac{C_{H_{1}}^{u} P}{\sqrt{\left|C_{H_{1}}^{u} P\right|^{2}+m_{h}^{2}}} h^{u}, \\
& H^{d}=\frac{m_{h}}{\sqrt{\left|C_{H_{1}}^{d} P\right|^{2}+m_{h}^{2}}} H_{d}^{0}-\frac{C_{H_{1}}^{d} P}{\sqrt{\left|C_{H_{1}}^{d} P\right|^{2}+m_{h}^{2}}} h^{d} .
\end{aligned}
$$

These contents of MSSM doublets can be also seen explicitly in the full mass matrix of the doublets

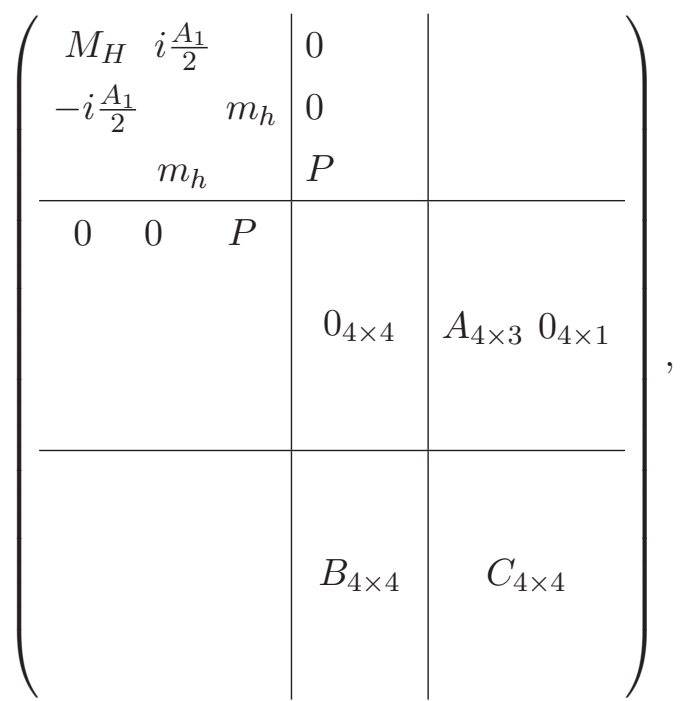

where both the rows and the columns are ordered as

$$
\left(H_{2}, h, \bar{h} ; H_{1}, D_{1}, D_{1}^{\prime}, \bar{\Delta} ; \Delta, D_{2}, D_{2}^{\prime}, \Phi\right) .
$$

It is clear that taking the DW solution $A_{1}=0$, a combination of $h^{d}, H_{1}^{d}, D_{1}^{d}, D_{1}^{\prime d}, \bar{\Delta}^{d}$ gives the massless eigenstate $H_{d}$ of the MSSM, and a combination of $h^{u}, H_{1}^{u}, D_{1}^{u}, D_{1}^{\prime u}, \bar{\Delta}^{u}$ and $\Phi^{u}$ gives $H_{u}$. 
The triplets are relevant for proton decay amplitudes and their treatments are different. The triplets which do not couple with the fermions can be integrated out to get effective masses for those triplets which couple with the fermions. We can integrate out the triplets in $h, \bar{h}, H_{2}$ in (4.3) (not in (4.7)) firstly. The result is generating an effective mass

$$
m_{H_{1}}^{\mathrm{eff}}=\left(\frac{A_{2}}{m_{h}}\right)^{2} \frac{P^{2}}{m_{2}}
$$

for the triplets in $H_{1}$ responsible for proton decay. To suppress proton decay mediated by the triplets in $H_{1}, m_{H_{1}}^{\text {eff }}$ needs to be enhanced. Taking the mass parameters $m_{h}$ and $m_{2}$ the same order as the GUT scale determined by the GUT breaking VEVs $\Phi_{1,2,3} \sim A_{2} \sim M_{G}$, a large VEV $P \sim 10 M_{G}$ is needed which may be realized through the Green-Shwarz mechanism [35-38]. Note that in the original DW mechanism, the effective triplet mass is $\frac{A_{2}^{2}}{m_{2}}$ whose enhancement needs a small $m_{2}$ which, being the mass of the doublets of $H_{2}$, leads to large splitting between the the doublets in $H_{2}$ and triplets in $H_{1,2}$, and the resultant threshold effect $[8,9]$ spoils gauge coupling unification. This problem is absent in the present model since the threshold effect is proportional to [39]

$$
\ln \frac{M_{G} \operatorname{Det}^{\prime}\left(M_{D}\right)}{\operatorname{Det}\left(M_{T}\right)}
$$

where $\operatorname{Det}^{\prime}\left(M_{D}\right)=\lim _{\epsilon \rightarrow 0} \frac{1}{\epsilon} \operatorname{Det}\left(M_{D}+\epsilon \hat{\mathrm{I}}_{4 \times 4}\right)[33,34]$. For a large $P,(4.10)$ is small so that gauge coupling unification is maintained.

The filter sector can be generalized beyond the superpotential (4.1) if the following terms

$$
m^{\prime} \bar{h}^{2}+m^{\prime \prime} \bar{h} H_{2}
$$

are added. They affect neither the massless eigenstates nor the threshold effects, provided a large $P$ is taken.

There are more comments to be added if a filter sector is useful in building GUT models. First, the singlet $P$ can be replaced by a $\mathbf{5 4}$ when it is needed. Second, the easiest way to construct the filter sector superpotential (4.2) is using a new $\mathbb{Z}_{2}$ symmetry with the only fields odd under this $\mathbb{Z}_{2}$ symmetry are $P, h, \bar{h}$ and $H_{2}$.

\section{The full model}

Having all the requirements given, we give a full model with DW mechanism to realize the DTS. We impose a $\mathbb{Z}_{12} \times \mathbb{Z}_{2}$ symmetry. The superfields and their transformation properties are summarized in table II.

The most general superpotential for the Higgs sector is

$$
W=W_{D W}+W_{S B}+W_{\text {filter }}^{\prime}+W_{D T}^{\prime}
$$

where $W_{D W}$ and $W_{S B}$ are given in (2.1) and (2.2), respectively,

$$
W_{\text {filter }}^{\prime}=W_{\text {filter }}+\bar{h} S^{\prime} h
$$




\begin{tabular}{|c|c|c|c|c|c|c|c|c|c|c|c|c|c|c|c|c|}
\hline & $H_{1}$ & $\bar{h}$ & $h$ & $H_{2}$ & $D_{1}$ & $D_{2}$ & $\bar{\Delta}$ & $\Delta$ & $\Phi$ & $A$ & $A^{\prime}$ & $A^{\prime \prime}$ & $S$ & $S^{\prime}$ & $P$ & $Y$ \\
\hline $\mathbb{Z}_{12}$ & 2 & 5 & -5 & 6 & 2 & -2 & 2 & -2 & 0 & -1 & -5 & 6 & 6 & 0 & 5 & 6 \\
\hline $\mathbb{Z}_{2}$ & 0 & 1 & 1 & 1 & 0 & 0 & 0 & 0 & 0 & 0 & 0 & 0 & 0 & 0 & 1 & 0 \\
\hline
\end{tabular}

Table 2. Fields and symmetric properties under $\mathbb{Z}_{12} \times \mathbb{Z}_{2}$ in the full model with $\mathbf{1 2 0}$ included. The charges for all the matter fields are $(-1,0)$ under this symmetry.

is the complete filter sector, and

$$
\begin{aligned}
W_{D T}^{\prime}= & \left(H_{1}+D_{1}+\bar{\Delta}\right) \Phi\left(\Delta+D_{2}\right) \\
& +m_{D} D_{1} D_{2}+m_{\Delta} \Delta \bar{\Delta}+D_{1} S^{\prime} D_{2}
\end{aligned}
$$

is the main doublet-triplet sector.

SUSY at high energy requires the F-flatness conditions. Among them only $F_{P}=0$ is automatic so that the VEV $P$ can take a value larger than the GUT scale through the Green-Schwarz mechanism [35-38], as the $\mathbb{Z}_{12} \times \mathbb{Z}_{2}$ are embed into U(1)'s. Since these $\mathrm{U}(1)$ 's are anomalous, the D-terms have the form

$$
D_{A}=-\xi+\sum Q_{i}\left|\phi_{i}\right|^{2}, \quad \xi=\frac{\operatorname{Tr} Q}{192 \pi^{2}} M_{\text {Planck }}^{2},
$$

where $Q_{i}$ is the $\mathrm{U}(1)$ charge of the scalar field $\phi_{i}$, thus the VEV of $P$ is related the Planck scale $M_{\text {Planck }}$. All the other VEVs are constrained by the F-flatness conditions and have solutions of the GUT scale values.

The present model (5.1) has the following consequences. First, it contains Higgs in 10, $\overline{\mathbf{1 2 6}}$ and $\mathbf{1 2 0}$ which transform the same way under the extra symmetry so that they can give the masses and mixing of the three generation matter particles through renormalizble Yukawa couplings. Second, it realizes naturally the DTS through the DW mechanism supplied by a filter sector. Third, proton decay amplitudes through dimension-five operators mediated by the Higgs triplets in $\mathbf{1 0}$ is suppressed without large threshold effect, although general suppressions of all proton decay amplitudes are not discussed which need structural constructions of models like is done in [32] and are beyond present study. Finally, we need to mention that from (4.8) that the up-type Higgs doublet of the MSSM has a large component from $h$ which does not couple with the matter fields, which might rise a difficulty in generating the large top quark mass whose Yukawa coupling is large. In a more realistic model, we need to modify the VEV of $P$ by a GUT-valued VEV of a $\mathrm{SO}(10)$ singlet or $\mathbf{5 4}$ to fix this problem. To suppress proton decay mediated by the color triplets in $H_{1}$, a small $m_{h}$ needs to be taken whose origin might be related to the seesaw scale $M_{\text {seesaw }} \sim \frac{M_{G U T}^{2}}{M_{\text {Planck }}}$ generated through the Green-Scwarz mechanism, meanwhile all the other proton decay amplitudes are also suppressed by $\frac{M_{\text {seesaw }}}{M_{G U T}}[40]$.

\section{Summary}

In the present work we have proposed a renormalizable SUSY SO(10) model of naturally realized DTS through the DW mechanism. A filter sector is introduced to forbid an unwanted 
coupling which spoils the DW mechanism. Proton decay mediated by the color-triplets in $H_{1}$ can be suppressed without spoiling gauge unification. However, a problem on the contents of the MSSM Higgs doublets which are insufficient to give top quark correct mass. This problem requires the present model to be further improved.

As in all renormalizable SUSY GUT models, the large representations used in the present model also bring in big contributions to the $\beta$-function of the GUT gauge coupling. Consequently, the GUT gauge coupling blows up quickly above the GUT scale. This non-perturbative problem may not be a serious one if we take the following picture. The universe was in the GUT symmetric phase at very high temperature in its early stage. As the universe was cooling down, phase transition happened and GUT symmetry was broken. Without knowing more details about what was happening during this phase transition, the non-perturbative behavior of the GUT gauge coupling above the GUT scale may not be a problem.

\section{A The full mass matrix for the weak doublets}

Denoting

$$
\begin{aligned}
m_{2,3}=m_{3,2} & =m_{h}+\frac{1}{2} \sqrt{\frac{3}{5}} S^{\prime}, \\
m_{4,7} & =\frac{\Phi_{2}}{\sqrt{10}}-\frac{\Phi_{3}}{2 \sqrt{5}}, \\
m_{5,9}=m_{9,5} & =m_{d}+\frac{1}{2} \sqrt{\frac{3}{5}} S^{\prime}, \\
m_{6,7} & =\frac{\Phi_{1}}{4 \sqrt{15}}-\frac{\Phi_{3}}{6 \sqrt{10}}, \\
m_{6,10} m_{10,6} & =m_{d}-\frac{S^{\prime}}{6 \sqrt{15}}+\frac{\sqrt{2} \Phi_{2}}{9}, \\
m_{7,4} & =-\frac{\Phi_{2}}{\sqrt{10}}-\frac{\Phi_{3}}{2 \sqrt{5}}, \\
m_{7,6} & =\frac{\Phi_{1}}{4 \sqrt{15}}+\frac{\Phi_{3}}{6 \sqrt{10}}, \\
m_{7,8} & =m_{\Delta}+\frac{\Phi_{2}}{15 \sqrt{2}}+\frac{\Phi_{3}}{30}, \\
m_{8,7} & =m_{\Delta}+\frac{\Phi_{2}}{15 \sqrt{2}}-\frac{\Phi_{3}}{30}, \\
m_{8,10} & =\frac{\Phi_{1}}{4 \sqrt{15}}-\frac{\Phi_{3}}{6 \sqrt{10}}, \\
m_{10,8} & =\frac{\Phi_{1}}{4 \sqrt{15}}+\frac{\Phi_{3}}{6 \sqrt{10}}, \\
m_{11} & =m_{\Phi}-\frac{1}{4} \sqrt{\frac{3}{5}} S^{\prime}+\frac{\Phi_{2}}{\sqrt{2}}+\frac{\Phi_{3}}{2},
\end{aligned}
$$

and ordering both the rows and the columns as

$$
\left(H_{2}, h, \bar{h} ; H_{1}, D_{1}, D_{1}^{\prime}, \bar{\Delta} ; \Delta, D_{2}, D_{2}^{\prime}, \Phi\right),
$$


the mass matrix for all the weak doublets in the full model can be given after the most general superpotential (5.1),

$$
\left(\begin{array}{ccccccccccc}
m_{2} & \frac{i A_{1}}{2} & 0 & 0 & 0 & 0 & 0 & 0 & 0 & 0 & 0 \\
-\frac{i A_{1}}{2} & 0 & m_{2,3} & 0 & 0 & 0 & 0 & 0 & 0 & 0 & 0 \\
0 & m_{3,2} & 0 & P & 0 & 0 & 0 & 0 & 0 & 0 & 0 \\
0 & 0 & P & 0 & 0 & 0 & 0 & m_{4,8} & -\frac{\Phi_{1}}{2} & -\frac{\Phi_{3}}{2 \sqrt{2}} & 0 \\
0 & 0 & 0 & 0 & 0 & 0 & 0 & \frac{\Phi_{3}}{4 \sqrt{30}} & m_{5,9} & \frac{\Phi_{3}}{6 \sqrt{3}} & 0 \\
0 & 0 & 0 & 0 & 0 & 0 & 0 & m_{6,8} & \frac{\Phi_{3}}{6 \sqrt{3}} & m_{6,10} & 0 \\
0 & 0 & 0 & 0 & 0 & 0 & 0 & m_{7,8} & \frac{\Phi_{3}}{4 \sqrt{30}} & m_{7,10} & 0 \\
0 & 0 & 0 & m_{8,4} & \frac{\Phi_{3}}{4 \sqrt{30}} & m_{8,6} & m_{8,7} & 0 & 0 & 0 & \frac{\overline{v_{R}}}{10} \\
0 & 0 & 0 & -\frac{\Phi_{1}}{2} & m_{9,5} & \frac{\Phi_{3}}{6 \sqrt{3}} & \frac{\Phi_{3}}{4 \sqrt{30}} & 0 & 0 & 0 & -\frac{\overline{v_{R}}}{2 \sqrt{30}} \\
0 & 0 & 0 & -\frac{\Phi_{3}}{2 \sqrt{2}} & \frac{\Phi_{3}}{6 \sqrt{3}} & m_{10,6} & m_{10,7} & 0 & 0 & 0 & -\frac{\sqrt{v_{R}}}{2 \sqrt{10}} \\
0 & 0 & 0 & -\frac{v_{R}}{\sqrt{5}} & -\frac{v_{R}}{2 \sqrt{30}} & -\frac{v_{R}}{2 \sqrt{10}} & \frac{v_{R}}{10} & 0 & 0 & 0 & m_{11}
\end{array}\right) .
$$

Open Access. This article is distributed under the terms of the Creative Commons Attribution License (CC-BY 4.0), which permits any use, distribution and reproduction in any medium, provided the original author(s) and source are credited.

\section{References}

[1] T.E. Clark, T.-K. Kuo and N. Nakagawa, A SO(10) supersymmetric grand unified theory, Phys. Lett. B 115 (1982) 26 [InSPIRE].

[2] C.S. Aulakh and R.N. Mohapatra, Implications of supersymmetric $\mathrm{SO}(10)$ grand unification, Phys. Rev. D 28 (1983) 217 [inSPIRE].

[3] K.S. Babu, I. Gogoladze and Z. Tavartkiladze, Missing partner mechanism in $\mathrm{SO}(10)$ grand unification, Phys. Lett. B 650 (2007) 49 [hep-ph/0612315] [INSPIRE].

[4] K.S. Babu, I. Gogoladze, P. Nath and R.M. Syed, Variety of $\mathrm{SO}(10)$ GUTs with natural doublet-triplet splitting via the missing partner mechanism, Phys. Rev. D 85 (2012) 075002 [arXiv:1112.5387] [INSPIRE].

[5] L. Du, X. Li and D.-X. Zhang, Proton decay in a supersymmetric $\mathrm{SO}(10)$ model with missing partner mechanism, JHEP 04 (2014) 027 [arXiv: 1312.1786] [INSPIRE].

[6] S. Dimopoulos and F. Wilczek, Incomplete multiplets in supersymmetric unified models, NSF-ITP-82-07, U.S.A. (1981) [INSPIRE].

[7] M. Srednicki, Supersymmetric grand unified theories and the early universe, Nucl. Phys. B 202 (1982) 327 [InSPIRE].

[8] K.S. Babu and S.M. Barr, Natural suppression of Higgsino mediated proton decay in supersymmetric SO(10), Phys. Rev. D 48 (1993) 5354 [hep-ph/9306242] [INSPIRE].

[9] K.S. Babu and S.M. Barr, Natural gauge hierarchy in SO(10), Phys. Rev. D 50 (1994) 3529 [hep-ph/9402291] [INSPIRE]. 
[10] D.-G. Lee and R.N. Mohapatra, Natural doublet-triplet splitting in supersymmetric $\mathrm{SO}(10)$ models, Phys. Lett. B 324 (1994) 376 [hep-ph/9310371] [INSPIRE].

[11] J. Hisano, H. Murayama and T. Yanagida, Double-triplet splitting in a supersymmetric SO(10) model without fine tuning, Phys. Rev. D 49 (1994) 4966 [InSPIRE].

[12] K.S. Babu and S.M. Barr, Supersymmetric SO(10) simplified, Phys. Rev. D 51 (1995) 2463 [hep-ph/9409285] [INSPIRE].

[13] Z. Berezhiani and Z. Tavartkiladze, More missing VEV mechanism in supersymmetric SO(10) model, Phys. Lett. B 409 (1997) 220 [hep-ph/9612232] [INSPIRE].

[14] S.M. Barr and S. Raby, Minimal SO(10) unification, Phys. Rev. Lett. 79 (1997) 4748 [hep-ph/9705366] [INSPIRE].

[15] Z. Chacko and R.N. Mohapatra, Economical doublet triplet splitting and strong suppression of proton decay in $\mathrm{SO}(10)$, Phys. Rev. D 59 (1999) 011702 [hep-ph/9808458] [INSPIRE].

[16] Z. Chacko and R.N. Mohapatra, A new doublet-triplet splitting mechanism for supersymmetric $\mathrm{SO}(10)$ and implications for fermion masses, Phys. Rev. Lett. 82 (1999) 2836 [hep-ph/9810315] [INSPIRE].

[17] K.S. Babu and S.M. Barr, Eliminating the D $=5$ proton decay operators from SUSY GUTs, Phys. Rev. D 65 (2002) 095009 [hep-ph/0201130] [InSPIRE].

[18] K.S. Babu, J.C. Pati and Z. Tavartkiladze, Constraining proton lifetime in $\mathrm{SO}(10)$ with stabilized doublet-triplet splitting, JHEP 06 (2010) 084 [arXiv:1003.2625] [INSPIRE].

[19] B. Bajc, A. Melfo, G. Senjanović and F. Vissani, Fermion mass relations in a supersymmetric SO(10) theory, Phys. Lett. B 634 (2006) 272 [hep-ph/0511352] [INSPIRE].

[20] S. Bertolini, M. Frigerio and M. Malinsky, Fermion masses in SUSY SO(10) with type-II seesaw: a non-minimal predictive scenario, Phys. Rev. D 70 (2004) 095002 [hep-ph/0406117] [INSPIRE].

[21] W.-M. Yang and Z.-G. Wang, Fermion masses and flavor mixing in a supersymmetric SO(10) model, Nucl. Phys. B 707 (2005) 87 [hep-ph/0406221] [INSPIRE].

[22] C.S. Aulakh and S.K. Garg, MSGUT: from bloom to doom, Nucl. Phys. B 757 (2006) 47 [hep-ph/0512224] [INSPIRE].

[23] W. Grimus and H. Kuhbock, Fermion masses and mixings in a renormalizable $\mathrm{SO}(10) \times Z_{2}$ GUT, Phys. Lett. B 643 (2006) 182 [hep-ph/0607197] [INSPIRE].

[24] M. Malinsky, Higgs sector of the next-to-minimal renormalizable SUSY SO(10), arXiv:0807.0591 [INSPIRE].

[25] L. Lavoura, H. Kuhbock and W. Grimus, Charged-fermion masses in $\mathrm{SO}(10)$ : analysis with scalars in $10+120$, Nucl. Phys. B 754 (2006) 1 [hep-ph/0603259] [INSPIRE].

[26] W. Grimus and H. Kuhbock, A renormalizable $\mathrm{SO}(10)$ GUT scenario with spontaneous CP-violation, Eur. Phys. J. C 51 (2007) 721 [hep-ph/0612132] [INSPIRE].

[27] C.S. Aulakh and S.K. Garg, The new minimal supersymmetric GUT: spectra, RG analysis and fermion fits, Nucl. Phys. B 857 (2012) 101 [arXiv:0807.0917] [INSPIRE].

[28] A.S. Joshipura, B.P. Kodrani and K.M. Patel, Fermion masses and mixings in a $\mu-\tau$ symmetric $\mathrm{SO}(10)$, Phys. Rev. D 79 (2009) 115017 [arXiv:0903.2161] [InSPIRE]. 
[29] G. Altarelli and G. Blankenburg, Different $\mathrm{SO}(10)$ paths to fermion masses and mixings, JHEP 03 (2011) 133 [arXiv: 1012.2697] [INSPIRE].

[30] A.S. Joshipura and K.M. Patel, Fermion masses in $\mathrm{SO}(10)$ models, Phys. Rev. D 83 (2011) 095002 [arXiv: 1102.5148] [INSPIRE].

[31] A. Dueck and W. Rodejohann, Fits to SO(10) grand unified models, JHEP 09 (2013) 024 [arXiv: 1306.4468] [INSPIRE].

[32] L. Du, X. Li and D.-X. Zhang, Connection between proton decay suppression and seesaw mechanism in supersymmetric $\mathrm{SO}(10)$ models, JHEP 10 (2014) 036 [arXiv:1406.2081] [INSPIRE].

[33] B. Bajc, A. Melfo, G. Senjanović and F. Vissani, The minimal supersymmetric grand unified theory. 1. Symmetry breaking and the particle spectrum, Phys. Rev. D 70 (2004) 035007 [hep-ph/0402122] [INSPIRE].

[34] T. Fukuyama, A. Ilakovac, T. Kikuchi, S. Meljanac and N. Okada, SO(10) group theory for the unified model building, J. Math. Phys. 46 (2005) 033505 [hep-ph/0405300] [INSPIRE].

[35] M.B. Green and J.H. Schwarz, Anomaly cancellation in supersymmetric D $=10$ gauge theory and superstring theory, Phys. Lett. B 149 (1984) 117 [INSPIRE].

[36] M. Dine, N. Seiberg and E. Witten, Fayet-Iliopoulos terms in string theory, Nucl. Phys. B 289 (1987) 589 [inSPIRE].

[37] J.J. Atick, L.J. Dixon and A. Sen, String calculation of Fayet-Iliopoulos D-terms in arbitrary supersymmetric compactifications, Nucl. Phys. B 292 (1987) 109 [InSPIRE].

[38] M. Dine, I. Ichinose and N. Seiberg, F-terms and D-terms in string theory, Nucl. Phys. B 293 (1987) 253 [inSPIRE].

[39] J.-H. Zheng and D.-X. Zhang, A renormalizable supersymmetric SU(5) model, JHEP 02 (2012) 046 [arXiv:1202.5072] [INSPIRE].

[40] X. Li and D.-X. Zhang, Proton decay suppression in a supersymmetric $\mathrm{SO}(10)$ model, arXiv: 1409.5233 [INSPIRE]. 